TITLE:

\title{
Two-dimensional spectroscopy for a two-dimensional rotator coupled to a Gaussian-Markovian noise bath
}

$\operatorname{AUTHOR}(S):$

Suzuki, Y; Tanimura, Y

\section{CITATION:}

Suzuki, Y ...[et al]. Two-dimensional spectroscopy for a two-dimensional rotator coupled to a Gaussian-Markovian noise bath. JOURNAL OF CHEMICAL PHYSICS 2003, 119(3): 16501660

ISSUE DATE:

2003-07-15

URL:

http://hdl.handle.net/2433/49970

\section{RIGHT:}

Copyright 2003 American Institute of Physics. This article may be downloaded for personal use only. Any other use requires prior permission of the author and the American Institute of Physics. 


\title{
Two-dimensional spectroscopy for a two-dimensional rotator coupled to a Gaussian-Markovian noise bath
}

\author{
Yoko Suzukia) and Yoshitaka Tanimura \\ Institute for Molecular Science, Myodaiji, Okazaki 444-8585, Japan
}

(Received 22 November 2002; accepted 9 April 2003)

\begin{abstract}
The dynamics of a system in the condensed phase are more clearly characterized by multitime correlation functions of physical observables than by two-time ones. We investigate a two-dimensional motion of a rigid rotator coupled to a Gaussian-Markovian harmonic oscillator bath to probe this issue. The analytical expression of a four-time correlation function of a dipole that is the observable of two-dimensional microwave or far-infrared spectroscopy is obtained from a generating functional approach. The spectra in the absence of damping are discrete and reveal transitions between eigenstates of the angular momentum quantized due to the cyclic boundary condition. For a weakly damped case, the two-dimensional spectrum predicts three echolike peaks corresponding to transition processes between the rotational energy levels, which cannot be observed in one-dimensional (linear-absorption) spectroscopy related to the two-time correlation function of the dipole [J. Phys. Soc. Jpn. 71, 2414 (2002)]. The two-dimensional spectra are more sensitive to the noise effects than the one-dimensional spectra. It is because the effects of the initial thermal distribution determine the profile of the continuous line shape in one-dimensional spectroscopy, while such thermal effects are canceled through the higher-order optical transition process in two-dimensional spectroscopy. If the rotator system is strongly coupled to the colored noise bath, the system exhibits one overdamped and other oscillatory motions. We observe peaks arising from interaction between these two modes in the two-dimensional spectra, which are difficult to distinguish in one-dimensional spectra. (c) 2003 American Institute of Physics.
\end{abstract}

[DOI: $10.1063 / 1.1578630]$

\section{INTRODUCTION}

Femtosecond nonlinear optical spectroscopies have proven to be valuable and versatile tools for investigating the structural and dynamical properties of a molecular system in the condensed phase. ${ }^{1}$ The properties of the system can be studied by measuring the change of the molecular dipole or Raman polarizability after exciting the system from an equilibrium state by applying external laser pulses. Due to the static inhomogeneity and/or dynamical relaxation, the observed spectral line shapes are broadened and it is not easy to analyze the mechanisms of molecular interactions, which govern many chemical processes including chemical reactions. Multidimensional laser spectroscopy, which is an analogue to multidimensional nuclear magnetic resonance spectroscopy, may overcome this problem by employing complex pulse sequences that suppress specific contributions to a spectrum selectivity arising from inter- and/or intramolecular couplings.

Theoretically, it has been shown that an appropriate multidimensional representation of the optical signal in Raman or IR measurements provides an interpretable depiction of the structural and dynamical properties in relation to the inhomogeneous distribution, ${ }^{2}$ the anharmonicity, ${ }^{3,4}$ intermolecular interactions (between atoms of different molecules) $)^{5-10}$ and/or intramolecular interactions (i.e., the interactions between the atoms of a molecule), ${ }^{11-17}$ relaxation mechanisms, ${ }^{18-20}$ and wave packet dynamics. ${ }^{21}$ Experimen-

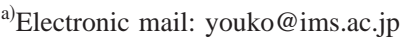

tally, some of these features have been studied by twodimensional Raman and IR spectroscopies. Fifth-order Raman spectroscopy has been performed on intermolecular vibrations of liquids $\mathrm{CS}_{2}$ (Refs. 22-24) by minimizing cascaded third-order (four-wave mixing) contributions. ${ }^{25,26}$ These experimental results indicate the existence of anharmonic vibrational modes. Nodal lines in the spectrum have been found in molecular dynamics simulation for liquid $\mathrm{CS}_{2}$ by Saito and Ohmine ${ }^{10}$ that may be explained by some contributions to the signal from the nonlinearity of the polarizability in addition to the anharmonicity. ${ }^{3}$ Experimental evidence for this effect has also been reported. ${ }^{24} \mathrm{~A}$ second-order IR signal carries the same information as the fifth-order Raman one, but due to the isotropy of liquids such measurements are impossible except for anisotropic conditions such as adsorbed molecule on the metallic surface. ${ }^{27}$ Therefore, the most applicable two-dimensional IR process is third order, which carries the equivalent information as seventhorder Raman processes. ${ }^{28-31}$ For either Raman or IR, the signal in this order is even weaker than fifth-order Raman if the vibrational modes are purely harmonic, because the signal is proportional to the square of the second-order nonlinear polarizability or dipole moment. ${ }^{2}$ To date, twodimensional (2D) IR measurements have been carried out primarily for intravibrational motions, where the anharmonicity of the potential or mode-couplings is reasonably strong. For example, the 2D Fourier plots of the three pulse vibrational echo technique applied to a dipeptide molecule illustrate the coupling between two amid-I modes. ${ }^{32}$ The de- 
gree of the correlation among vibrational modes in the conformational fluctuations of peptides ${ }^{16,33,34}$ and small molecules ${ }^{35}$ have also been investigated by 2D IR spectroscopy. In parallel with these investigations, researchers have explored two-dimensional spectroscopy in a broader context; second harmonic generation, ${ }^{36}$ DOVE spectroscopy, ${ }^{37,38}$ combination of IR and optical sources, ${ }^{12,27}$ etc. Such experimental efforts provide further stimulus for theoretical studies. $^{39-43}$

The above-mentioned 2D spectroscopies have been utilized to investigate the vibrational motions arising from intra- and intermolecular interactions. In the short time range, typically from femtoseconds to picoseconds, collective motions play a central role. In the long time range, typically from a few picoseconds to nanoseconds depending on the molecular size and on the viscosity, the orientational diffusion of a single molecule becomes very important. The picture of a single molecule librating in the potential well made up by the surrounding molecules is acceptable for a slow solute in a fast solvent. For understanding molecular dynamics, rotational motions are as important as vibrational ones. $^{44-48}$ It has been shown that the rotational relaxation plays a major role in dielectric absorption ${ }^{49}$ and dispersion, the IR, far-IR, or rotational-Raman spectra ${ }^{50,51}$ of solutions. But, due to the inhomogeneity and damping, experiments have had difficulty observing some important features such as quantum effects, e.g., rotational-energy discretization and transition between energy levels where energy gaps depend on the quantum number, which cannot be seen in the harmonic oscillator case. In this paper, we demonstrate that we can extract more information for rotational motions by utilizing two-dimensional spectroscopy.

To obtain an analytical expression for the third-order far-IR or microwave response function, we use the generating functional for a quantal two-dimensional rotator coupled to a heat-bath. This approach has been used previously to study linear absorption spectra of the damped rotator, taking into account noise correlation effects. ${ }^{52}$ The present study is an extension of this work. In Sec. II, we present the model Hamiltonian and the analytical expression of the fourth-order correlation function for the dipole moment for the case of Gaussian-Markovian noise, as derived from the generating functional obtained previously. ${ }^{52}$ The calculational details are shown in Appendix A. The numerical results and their discussions are presented in Sec. III.

\section{RESPONSE FUNCTION FOR OPTICAL PROCESSES}

We consider the Hamiltonian of the two-dimensional rotator system coupled to an environment in the form

$$
\hat{H}=\frac{L^{2}}{2 \mu}+\sum_{i}\left[\frac{\hat{p}_{i}^{2}}{2 m_{i}}+\frac{m_{i} \omega_{i}^{2}}{2}\left(\hat{q}_{i}-\frac{c_{i} \theta}{m_{i} \omega_{i}^{2}}\right)^{2}\right] .
$$

Here, $L$ and $\theta$ are the angular momentum defined by $L$ $\equiv(\hbar / i) \partial / \partial \theta$ and the angular coordinate, respectively. The angular coordinate is $-\pi \leqslant \theta<\pi$ with $\theta=-\pi$ and $\theta=\pi$ identified. The operators $\hat{q}_{i}$ and $\hat{p}_{i}$ and the parameters $m_{i}$ and $\omega_{i}$ denote the coordinate, conjugated momentum, mass, and frequency of the $i$ th bath oscillator, where the bath operators $\hat{q}_{i}$ and $\hat{p}_{i}$ commutate with the system operators $\theta$ and $L$. The coupling constant between the system and the $i$ th bath oscillator is given by $c_{i}$. Note that the original rotational symmetry of the rotator recovers after tracing out the bath degrees of freedom owing to the properties of the Gaussian integration. In the classical limit, we can reduce to the classical Langevin equation for the rotational motion from Eq. (2.1). ${ }^{52}$

We consider the optical response of the rigid rotator. If the system interacts with a laser field $E(t)$, the effective Hamiltonian including the laser interaction is given by $\hat{H}_{\text {eff }}$ $=\hat{H}-E(t) d_{0} \cos \theta$, where $d_{0}$ is the intensity of the dipole moment. We assume the system first interacts with $N$ pulses for the $N$ th-order optical process and the last pulse $E_{T}(t)$ is the probe to detect the rotational state. Since the even-order response vanishes due to rotational symmetry of the model, we study the odd-order responses. The laser pulses are assumed to be impulsive and are configured for the (i) firstand (ii) third-order processes as

$$
\text { (i) } \begin{aligned}
E_{1}(t) & =\delta(t), \quad E_{T}(t)=\delta\left(t-T_{1}\right), \\
E_{1}(t) & =\delta(t), \quad E_{2}(t)=\delta\left(t-T_{1}\right), \\
E_{3}(t) & =\delta\left(t-T_{1}-T_{2}\right), \\
E_{T}(t) & =\delta\left(t-T_{1}-T_{2}-T_{3}\right) .
\end{aligned}
$$

The optical signals are expressed by the $N$ th-order response functions as $I^{(N)}\left(T_{1}, T_{2}, \ldots, T_{N}\right) \propto R^{(N)}\left(T_{N}, \ldots, T_{2}, T_{1}\right)$, which are the $(N+1)$-time correlation functions of the dipole moment $\hat{d}=d_{0} \cos \theta$ given in terms of ${ }^{1}$

$$
\begin{aligned}
& R^{(N)}\left(T_{N}, \ldots, T_{2}, T_{1}\right) \\
&=\left(\frac{i}{\hbar}\right)^{N} d_{0}^{N+1}\left\langle\left[\left[\left[\cdots\left[\cos \theta\left(t_{0}\right), \cos \theta\left(t_{1}\right)\right], \ldots\right],\right.\right.\right. \\
&\left.\left.\left.\cos \theta\left(t_{N-1}\right)\right], \cos \theta\left(t_{N}\right)\right]\right\rangle,
\end{aligned}
$$

where we set $t_{i}=T_{1}+T_{2}+\cdots+T_{N-i}$ (for $i=0,1, \ldots, N$ $-1)$ and $t_{N}=0$. Here, $\cos \theta(t) \equiv e^{i \hat{H} t / \hbar} \cos \theta e^{-i \hat{H} t / \hbar}$ and $\langle\cdots\rangle$ means the expectation value of "..." defined by $\langle\cdots\rangle=\operatorname{Tr}\left(e^{-\beta \hat{H}} \cdots\right) / \operatorname{Tr} e^{-\beta \hat{H}}$ in which $\beta$ is the inverse temperature. Note that Eq. (2.4) corresponds to the $(2 \mathrm{~N}$ $+1)$ th-order off-resonant Raman response by the replacement of $\hat{d}$ with the polarizability $\alpha{ }^{1,2}$

The $N$ th-order response functions are derived from the generating functional, as shown in Appendix A. The firstorder response was studied in Refs. 52 and 53. The thirdorder response function for $T_{1}, T_{2}, T_{3}>0$ (i.e., $t_{0}>t_{1}>t_{2}$ $>t_{3}$ ) derived from Eq. (A12) is given by

$$
\begin{aligned}
R^{(3)}\left(T_{3}, T_{2}, T_{1}\right) \\
=\left(\frac{i}{\hbar}\right)^{3}\left\langle\left[\left[\left[\cos \theta\left(t_{0}\right), \cos \theta\left(t_{1}\right)\right], \cos \theta\left(t_{2}\right)\right], \cos \theta\left(t_{3}\right)\right]\right\rangle \\
=-\frac{1}{\Lambda \hbar^{3}}\left(R_{A}^{(3)}\left(T_{3}, T_{2}, T_{1}\right)+R_{B}^{(3)}\left(T_{3}, T_{2}, T_{1}\right)\right. \\
\left.\quad+R_{C}^{(3)}\left(T_{3}, T_{2}, T_{1}\right)\right),
\end{aligned}
$$


where $\Lambda=\Sigma_{l} e^{-\mu^{\prime}(2 \pi l)^{2} /\left(2 \beta \hbar^{2}\right)}$ with $\mu^{\prime}=\mu+\Sigma_{j} c_{j}^{2} /\left(m_{j} \omega_{j}^{4}\right)$. Here, the expression of the functions $R_{A}^{(3)}, R_{B}^{(3)}$, and $R_{C}^{(3)}$ are given in Appendix B. We consider the two-dimensional profile of the third-order response by setting $T_{2}=0$. Then Eq. (2.5) reduces to

$$
\begin{aligned}
R^{(3)}\left(T_{3}, T_{2}=0, T_{1}\right) & \\
= & -\frac{1}{\Lambda \hbar^{3}}\left(R_{A}^{(3)}\left(T_{3}, T_{2}=0, T_{1}\right)+R_{B}^{(3)}\left(T_{3}, T_{2}=0, T_{1}\right)\right. \\
& \left.\quad+R_{C}^{(3)}\left(T_{3}, T_{2}=0, T_{1}\right)\right),
\end{aligned}
$$

where

$$
\begin{aligned}
R_{A}^{(3)}( & \left.T_{3}, T_{2}=0, T_{1}\right) \\
= & R_{B}^{(3)}\left(T_{3}, T_{2}=0, T_{1}\right) \\
= & \sum_{l}\left\{\exp \left(-\frac{\mu^{\prime}(2 \pi l)^{2}}{2 \beta \hbar^{2}}\right) \cosh \left(\frac{2 \pi l}{\beta \hbar}\left(T_{1}+T_{3}\right)\right)\right\} \\
& \times \exp \left[-i \hbar \bar{K}_{0}^{(++)}\left(T_{1}+T_{3}\right)\right] \\
& \times\left[\sin \left(\frac{\hbar}{2} K_{0}^{(+-)}\left(T_{3}\right)\right)\right]^{2} \sin \left(\frac{\hbar}{2} K_{0}^{(+-)}\left(T_{1}+T_{3}\right)\right),
\end{aligned}
$$

$$
\begin{aligned}
R_{C}^{(3)}\left(T_{3}, T_{2}=0, T_{1}\right) \\
=\sum_{l}\left\{\exp \left(-\frac{\mu^{\prime}(2 \pi l)^{2}}{2 \beta \hbar^{2}}\right) \cosh \left(\frac{2 \pi l}{\beta \hbar}\left(-T_{1}+T_{3}\right)\right)\right\} \\
\quad \times \exp \left[i \hbar \left(-2 \bar{K}_{0}^{(++)}\left(T_{1}\right)-2 \bar{K}_{0}^{(++)}\left(T_{3}\right)\right.\right. \\
\left.\left.\quad+\bar{K}_{0}^{(++)}\left(T_{1}+T_{3}\right)\right)\right]\left[\sin \left(\frac{\hbar}{2} K_{0}^{(+-)}\left(T_{3}\right)\right)\right]^{2} \\
\quad \times \sin \left[\frac{\hbar}{2}\left(K_{0}^{(+-)}\left(T_{1}+T_{3}\right)-2 K_{0}^{(+-)}\left(T_{1}\right)\right)\right]
\end{aligned}
$$

The function $K_{0}^{(+-)}(t)$ is denoted in the Laplace representation as

$$
K_{0}^{(+-)}[z]=\frac{1}{\mu z^{2}+\mu \hat{\gamma}[z] z},
$$

where $\hat{\gamma}[z]$ is the Laplace transform of the mass independent damping kernel described as $\hat{\gamma}[z] \equiv \Sigma_{i} c_{i}^{2} z /\left[\mu m_{i} \omega_{i}^{2}\left(z^{2}\right.\right.$ $\left.\left.+\omega_{i}^{2}\right)\right]$. The function $\bar{K}_{0}^{(++)}(t)$ is expressed as

$$
\bar{K}_{0}^{(++)}(t) \equiv K_{0}^{(++)}(t)-K_{0}^{(++)}(0),
$$

where the Laplace representation of $K_{0}^{(++)}(t)$ is given by

$$
\begin{aligned}
K_{0}^{(++)}[z]= & \sum_{n=-\infty}^{\infty} e^{-i \nu_{n} 0^{+}}\left[\frac { i } { 2 } \left(\frac{K_{0}^{(+-)}\left[\nu_{n}\right]}{z+\nu_{n}}\right.\right. \\
& \left.\left.+\frac{K_{0}^{(+-)}\left[-\nu_{n}\right]}{z-\nu_{n}}\right)-\frac{i z K_{0}^{(+-)}[z]}{z^{2}-\nu_{n}^{2}}\right],
\end{aligned}
$$

with $\nu_{n} \equiv 2 \pi n /(\beta \hbar)$. In order to deal with dissipation, we introduce the spectral density of the environmental coupling
$I(\omega) \equiv \pi \Sigma_{i} c_{i}^{2} /\left(2 m_{i} \omega_{i}\right) \delta\left(\omega-\omega_{i}\right)$ and we regard $I(\omega)$ as a smooth function of $\omega$. With the aid of the spectral density, the heat bath is characterized by the mass independent damping kernel $\gamma(t) \equiv \int_{0}^{\infty} d \omega 2 I(\omega) \cos (\omega t) /(\pi \mu \omega)$, which implies the noise correlation function. In this paper, we assume Gaussian-Markovian noise, $\gamma(t)=\gamma \omega_{D} e^{-\omega_{D} t}$ with $I(\omega)$ $=\mu \omega \gamma \omega_{D}^{2} /\left(\omega_{D}^{2}+\omega^{2}\right)$, where $\gamma$ and $\omega_{D}$ correspond to the damping strength and the inverse correlation time of the fluctuations. ${ }^{54}$ We note that such a dissipation reduces to Gaussian-white noise in the limit $\omega_{D} \rightarrow \infty$. For GaussianMarkovian noise, the third-order response function Eq. (2.6) is expressed in terms of the functions

$$
\begin{aligned}
\bar{K}_{0}^{(++)}(t)= & \frac{i}{\beta \hbar \mu} \sum_{l=1}^{\infty} \frac{e^{-\nu_{l} t}-1}{\nu_{l}} \frac{2 \gamma \omega_{D}^{2}}{\left(\nu_{l}^{2}+\gamma \omega_{D}\right)^{2}-\nu_{l}^{2} \omega_{D}^{2}} \\
& -\frac{i t}{\beta \hbar \mu \gamma}+\frac{i}{4 \mu \zeta \gamma \omega_{D}}\left[\left(\frac{\omega_{D}}{2}+\zeta\right)^{2}\right. \\
& \times\left(1-e^{-\left(\omega_{D} / 2-\zeta\right) t}\right) \cot \left[\frac{\beta \hbar\left(\omega_{D}-2 \zeta\right)}{4}\right] \\
& -\left(\frac{\omega_{D}}{2}-\zeta\right)^{2}\left(1-e^{-\left(\omega_{D} / 2+\zeta\right) t}\right) \\
& \left.\times \cot \left[\frac{\beta \hbar\left(\omega_{D}+2 \zeta\right)}{4}\right]\right], \\
K_{0}^{(+-)}(t)= & \frac{1}{\mu \gamma}+\frac{e^{-\left(\omega_{D} / 2\right) t}}{\mu \zeta}\left(1-\frac{\omega_{D}}{2 \gamma}\right) \sinh (\zeta t) \\
& -\frac{e^{-\left(\omega_{D} / 2\right) t} \cosh (\zeta t),}{\mu \gamma}
\end{aligned}
$$

where

$$
\zeta=\frac{\omega_{D}}{2} \sqrt{1-\frac{4 \gamma}{\omega_{D}}} .
$$

Putting $\gamma=0$ into Eq. (2.6), we obtain the third-order response of the rotator without damping,

$R^{(3)}\left(T_{3}, T_{2}=0, T_{1} ; \gamma=0\right)$

$$
\begin{aligned}
= & -\frac{1}{\Lambda_{0} \hbar^{3}}\left(R_{A 0}^{(3)}\left(T_{3}, T_{2}=0, T_{1}\right)+R_{B 0}^{(3)}\left(T_{3}, T_{2}=0, T_{1}\right)\right. \\
& \left.+R_{C 0}^{(3)}\left(T_{3}, T_{2}=0, T_{1}\right)\right),
\end{aligned}
$$

where $\Lambda_{0}$ is defined as $\Lambda_{0}=\Sigma_{l} e^{-\beta E_{l}}$ and $R_{A 0}, R_{B 0}$, and $R_{C 0}$ are derived from the substitution $\gamma=0$ into $R_{A}, R_{B}$, and $R_{C}$, and are expressed as

$$
\begin{aligned}
R_{A 0}^{(3)}\left(T_{3}, T_{2}\right. & \left.=0, T_{1}\right)=R_{B 0}^{(3)}\left(T_{3}, T_{2}=0, T_{1}\right) \\
= & \sum_{l}\left(\frac{e^{-\beta E_{l}-e^{\beta E_{l+1}}}}{8}\right)\left(2 \sin \left[\frac{\hbar}{2 \mu}\left[(2 l+1)\left(T_{1}+T_{3}\right)\right]\right]\right. \\
& -\sin \left[\frac{\hbar}{2 \mu}\left[(2 l+1) T_{1}+(2 l+3) T_{3}\right]\right] \\
& -\sin \left[\frac{\hbar}{2 \mu}\left[(2 l+1) T_{1}+(2 l-1) T_{3}\right]\right)
\end{aligned}
$$




$$
\begin{aligned}
R_{C 0}^{(3)}\left(T_{3}, T_{2}=0, T_{1}\right) & \\
= & -\sum_{l}\left(\frac{e^{-\beta E_{l}-e^{\beta E_{l+1}}}}{8}\right)\left(2 \sin \left[\frac{\hbar}{2 \mu}\left[(2 l+1)\left(T_{1}-T_{3}\right)\right]\right]\right. \\
& -\sin \left[\frac{\hbar}{2 \mu}\left[(2 l+1) T_{1}-(2 l+3) T_{3}\right]\right] \\
& \left.-\sin \left[\frac{\hbar}{2 \mu}\left[(2 l+1) T_{1}-(2 l-1) T_{3}\right]\right]\right) .
\end{aligned}
$$

\section{NUMERICAL RESULT}

In this section, we calculate the third-order far-IR or microwave response functions for the rigid rotator coupled to a bath with Gaussian-Markovian noise using Eqs. (2.5)-(2.8). We study a frequency-domain two-dimensional signal that is the absolute value of the two-dimensional Fourier transform of the third-order response at $T_{2}=0$ [i.e., $I^{(3)}\left(\omega_{3}, \omega_{1}\right)$ $\left.=\left|\bar{I}^{(3)}\left(\omega_{3}, \omega_{1}\right)\right|\right]$,

$$
\begin{aligned}
\bar{I}^{(3)}\left(\omega_{3}, \omega_{1}\right)= & \int_{0}^{\infty} d T_{1} \int_{0}^{\infty} d T_{3} e^{i \omega_{1} T_{1}+i \omega_{3} T_{3}} \\
& \times R^{(3)}\left(T_{3}, T_{2}=0, T_{1}\right) .
\end{aligned}
$$

As will be shown in the following, 2D spectroscopy gives more information on the system dynamics than the onedimensional (1D) spectroscopy presented in Refs. 52 and 53. In 1D absorption spectra, a spectral line for the damped rotator exhibits a continuous band whose width depends on the temperature, the damping strength and the cutoff frequency; whereas, in 2D spectroscopy, a signal for the damped rotator shows the transition between discrete rotational energy levels that cannot be observed in 1D spectra.

For a demonstration purpose, we choose $\mu=2 \times 10^{-47}$ $\mathrm{kg} \mathrm{m}^{2}$, which is the value for the rotation motion of methyl group $\left(-\mathrm{CH}_{3}\right)$. Hereafter, we employed the parameter $\alpha$ $\equiv \hbar /(2 \mu)=2.6 \mathrm{THz}$. The heat bath corresponds to the nonpolar solvent whose effects are characterized by a GaussianMarkovian damping kernel.

In Fig. 1, we plot the 2D signal $I^{(3)}\left(\omega_{3}, \omega_{1}\right)$ for a free rotator at (a) $T=1 \mathrm{~K}$ and (b) $T=100 \mathrm{~K}$ by using Eqs. (2.15) and (3.1). The peaks rise at $\left(\omega_{1}, \omega_{3}\right)=((2 l+1) \alpha, \pm(2 l$ $+1) \alpha), \quad((2 l+1) \alpha, \pm(2 l+3) \alpha), \quad$ and $\quad((2 l+1) \alpha, \pm(2 l$ $-1) \alpha)(l=0, \pm 1, \pm 2, \ldots)$. The peak width is zero and the area of the circle at each peak in Fig. 1 represents the peak intensity.

The position of the peaks can be understood from double-sided Feynman diagrams. Figure 2 shows some of the double-sided Feynman diagrams for $R^{(3)}\left(T_{3}, T_{2}, T_{1}\right)$. In these diagrams, the upper horizontal line presents the time evolution of the left-hand side wave function (ket) whereas the lower represents the right-hand side (bra). We assume the system is initially in the ground state denoted by $|l\rangle\langle l|$, where $|l\rangle$ is an eigenstates of $L$ denoted by $L|l\rangle=\hbar l|l\rangle$. The arrows at the time $t-t_{I}=0, T_{1}$, and $T_{1}+T_{2}$ stand for the interactions with the radiation field and the arrow at $T_{1}$ $+T_{2}+T_{3}$ stands for the last probe field. An arrow pointing to (starting from) an upper horizontal line leads to the transition from $|l\rangle$ to $|l+1\rangle(|l-1\rangle)$, whereas an arrow pointing to

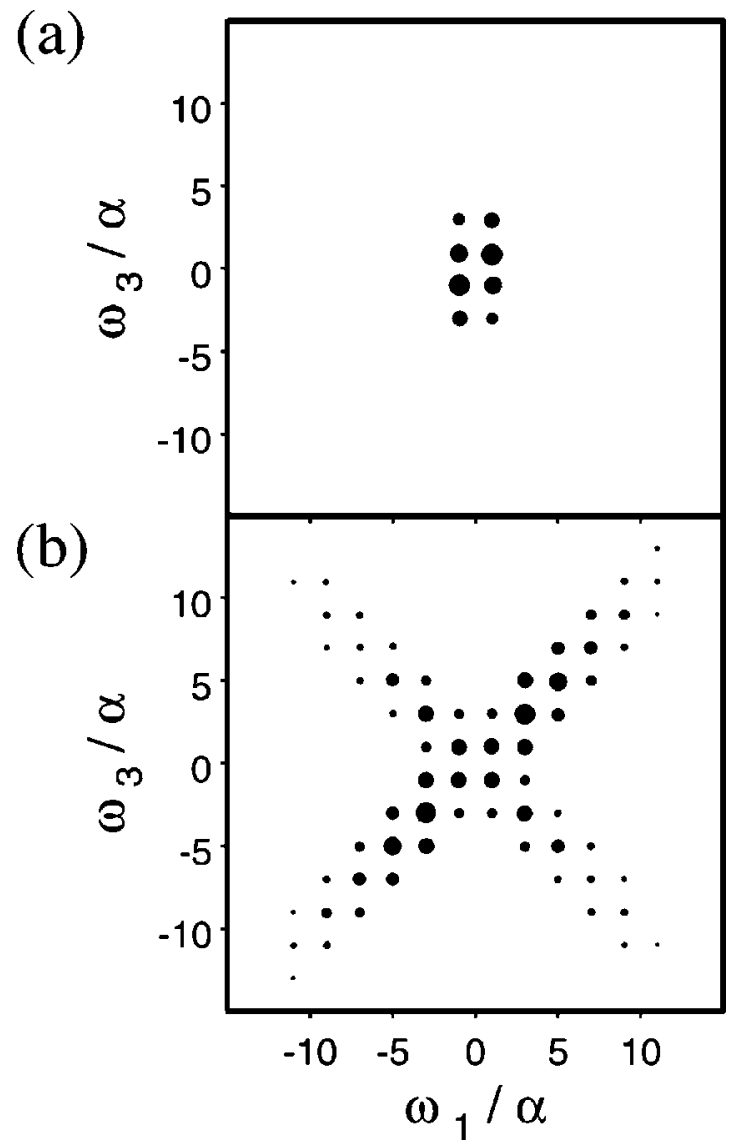

FIG. 1. Two-dimensional signal $I^{(3)}\left(\omega_{3}, \omega_{1}\right)$ for a free rotator at (a) $T=1 \mathrm{~K}$ and (b) $T=100 \mathrm{~K}$.

(starting from) a lower horizontal line assigned to the transition from $|l\rangle$ to $|l-1\rangle(|l+1\rangle)$. By putting each arrow to the upper or lower horizontal line, we have 96 diagrams for $R^{(3)}\left(T_{3}, T_{2}, T_{1}\right)$, where two arrows point to the ladder diagram and two arrows start from it. In the present case $T_{2}$ is set to be zero.

If the state during the period $T_{1}$ and the state during $T_{3}$ are denoted by $\left|l_{1}\right\rangle\left\langle l_{1}^{\prime}\right|$ and $\left|l_{3}\right\rangle\left\langle l_{3}^{\prime}\right|$, we obtain the factor $e^{-i \alpha \varphi_{13}\left(T_{1}, T_{3}\right)} \equiv e^{-i\left[\left(E_{l_{1}}-E_{l_{1}^{\prime}}\right) T_{1}+\left(E_{l_{3}}-E_{l_{3}^{\prime}}\right) T_{3}\right] / \hbar}$ with $E_{l}=\hbar \alpha l^{2}$, where the state $\left|l_{I}\right\rangle\left\langle l_{i}^{\prime}\right|(i=1,3)$ for $l_{I} \neq l_{i}^{\prime}$ and that for $l_{I}$ $=l_{i}^{\prime}$ refer to the coherence between states and the population state, respectively. By calculating $\varphi_{13}$ from Fig. 2, we can assign the peak positions at (a) $(-(2 l+1) \alpha,-(2 l+1) \alpha)$,

(a)

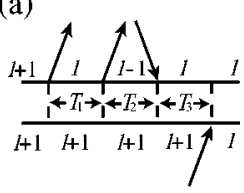

(d)

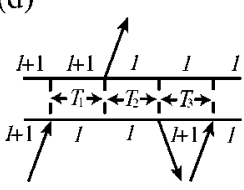

FIG. 2. Examples $R^{(7)}\left(T_{3}, T_{2}, T_{1}\right)$. (b)

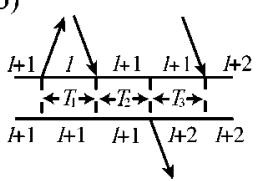

(e)

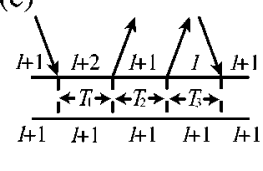

(c)

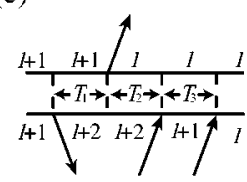

(f)

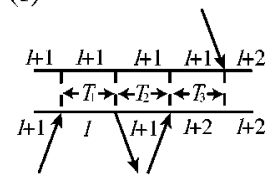


(a)

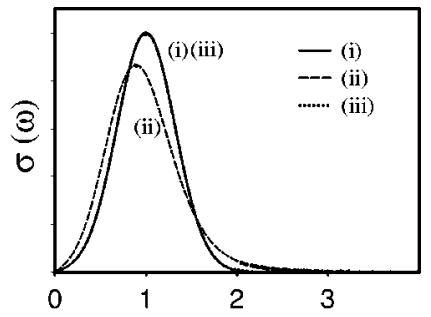

(b)

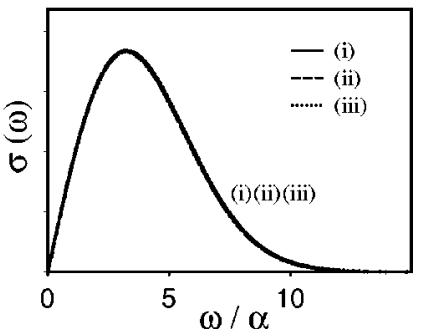

FIG. 3. Absorption spectra for the damped rotator at the temperature (a) $T$ $=1 \mathrm{~K}$ and (b) $T=100 \mathrm{~K}$. The damping strength and the cutoff frequency are set as (i) $\gamma=1.3 \times 10^{6} \mathrm{~Hz}, \omega_{D}=\infty$ (solid line), (ii) $\gamma=1.3 \times 10^{11} \mathrm{~Hz}, \omega_{D}$ $=\infty$ (dashed line), and (iii) $\gamma=1.3 \times 10^{11} \mathrm{~Hz}, \omega_{D}=0.1 \gamma$ (dotted line).

(b) $\quad(-(2 l+1) \alpha,-(2 l+3) \alpha), \quad$ (c) $\quad(-(2 l+3) \alpha,-(2 l$ $+1) \alpha),(\mathrm{d})((2 l+1) \alpha,-(2 l+1) \alpha),(\mathrm{e})((2 l+3) \alpha,-(2 l$ $+1) \alpha)$, and (f) $((2 l+1) \alpha,-(2 l+3) \alpha)(l=0, \pm 1, \ldots)$ to the diagrams (a)-(f) in Fig. 2, respectively.

From Eqs. (2.15)-(2.17), we see that the peak intensities depend on the initial thermal distribution. Since the initial thermal distribution for the initial state $|l\rangle\langle l|$ is given by $e^{-\beta \hbar \alpha l^{2}}$, the distribution of the peaks spread to the high frequency region with the increase in temperature, as shown in Figs. 1(a) and 1(b).

Let us consider the case of the weakly damped rotator $(\gamma<\alpha)$. Figure 3 shows the absorption spectra at the temperature (a) $T=1 \mathrm{~K}$ and (b) $T=100 \mathrm{~K}$ for the damping strength and the cutoff frequency set as (i) $\gamma=1.3 \times 10^{6} \mathrm{~Hz}$, $\omega_{D}=\infty$ (solid line), (ii) $\gamma=1.3 \times 10^{11} \mathrm{~Hz}, \omega_{D}=\infty$ (dashed line), and (iii) $\gamma=1.3 \times 10^{11} \mathrm{~Hz}, \omega_{D}=0.1 \gamma$ (dotted line). As can be seen from Fig. 3, the absorption spectra are not very sensitive to the noise effect represented by $\gamma$ and $\omega_{D}$ : the solid line agrees with the dotted one in Fig. 3(a), and the three lines (solid, dashed and dotted lines) in Fig. 3(b) cannot be distinguished.

Figure 4 shows the 2D signal $I^{(3)}\left(\omega_{3}, \omega_{1}\right)$ for same set of parameters in Figs. 3(i) -3(iii) at different temperatures (a) $T=1 \mathrm{~K}$ and (b) $T=100 \mathrm{~K}$. The noise effect is clearer in the 2D signal than in the 1D signal. In Fig. 4, the signals have peaks along $\omega_{1}=-\omega_{3}$ and $\omega_{1}=-\omega_{3} \pm \alpha$ except Fig. 4(b-ii), where the peaks are broadened and appear as a line of peaks along $\omega_{1}=-\omega_{3}$. Hereafter we will use the term "an antidiagonal peak line" to refer to the peaks along $\omega_{1}$ $=-\omega_{3}+$ (constant).

The peaks in Fig. 4 are explained from the diagrams given in Figs. 2(d)-2(f). For the damped rotator, the diagram in Figs. 2(a) -2 (c) and Figs. 2(d) -2 (f) give rise to the factors $\Sigma_{l} \rho_{l+1} e^{i \hbar l\left(T_{1}+T_{3}\right) / \mu}$ and $\Sigma_{l} \rho_{l+1} e^{i \hbar l\left(-T_{1}+T_{3}\right) / \mu}$, respectively, where $l$ implies the angular momentum and $\rho_{l}$ $\equiv e^{-\beta \hbar^{2} l^{2} /(2 \mu)}$. Since the linear coupling between the heat bath and the system changes the moment of inertia $\mu$ to $\mu^{\prime}$ (a)

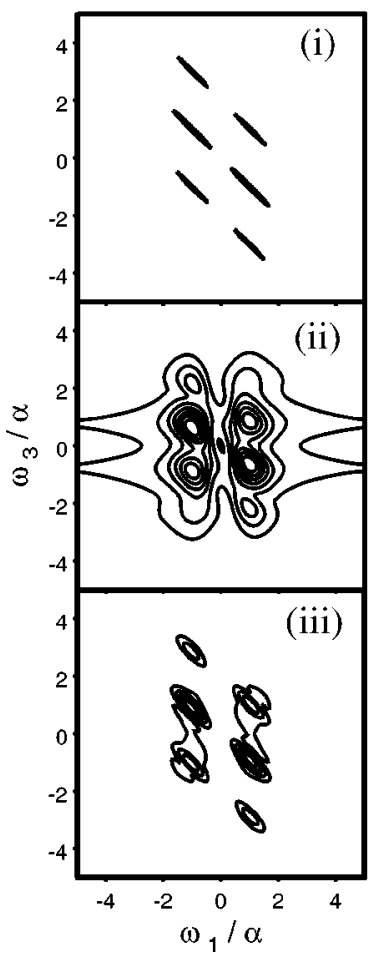

(b)

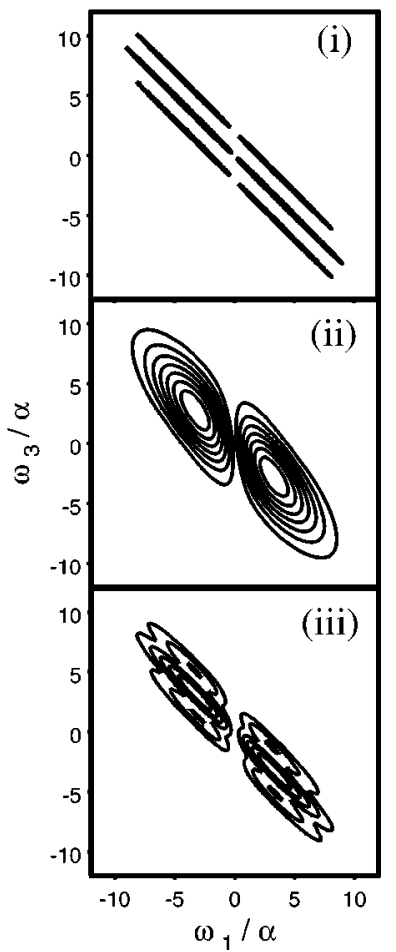

FIG. 4. Two-dimensional signal $I^{(3)}\left(\omega_{3}, \omega_{1}\right)$ for a weakly damped rotator at (a) $T=1 \mathrm{~K}$ (left column) and (b) $T=100 \mathrm{~K}$ (right column). The damping parameters are set as (i) $\gamma=1.3 \times 10^{6} \mathrm{~Hz}, \omega_{D}=\infty$, (ii) $\gamma=1.3 \times 10^{11} \mathrm{~Hz}$, $\omega_{D}=\infty$, and (iii) $\gamma=1.3 \times 10^{11} \mathrm{~Hz}, \omega_{D}=0.1 \gamma$.

being infinity in the Gaussian-Markovian noise, the $l$ dependence of the initial distribution function $\rho_{l}$ becomes infinitesimal. After summing over $l$ for $T_{1}, T_{3}>0$, the diagrams (d)-(f) in Fig. 2 lead the echolike tails along the diagonal $T_{1}=T_{3}$ in the $2 \mathrm{D}$ time-domain signal which give rise to the three "antidiagonal peak lines" in frequency domain as shown in Fig. 4, whereas the contributions of the diagrams in Figs. 2(a)-2(c) are small due to the cancellation between the terms $e^{i \hbar l\left(T_{1}+T_{3}\right) / \mu}(l=0, \pm 1, \ldots)$.

The diagrams in Figs. 2(d)-2(f) give the additional phases that are independent of the angular momentum $l$; (d) $e^{2 i \alpha\left(-T_{1}+T_{3}\right)}$, (e) $e^{2 i \alpha\left(-3 T_{1}+T_{3}\right)}$, and (f) $e^{2 i \alpha\left(-T_{1}+3 T_{3}\right)}$. They induce the $\omega_{3}$-axis intercepts in the contour plot of the 2D signal. Figures 2(d), 2(e), and 2(f) correspond to the peaks along $\omega_{3}=-\omega_{1}, \omega_{3}=-\omega_{1}+2 \alpha$ and $\omega_{3}=-\omega_{1}-2 \alpha$. The three "antidiagonal peak lines" in the 2D spectrum are a characteristic feature of the quantum rotator system.

We note that, for the damped Brownian rotator, information about the energy gap between the rotational level cannot be obtained in the $1 \mathrm{D}$ spectra. The $1 \mathrm{D}$ spectrum reveals a continuous broad peak that reflects the initial thermal distribution, because the system-bath coupling suppresses the effect of the energy discretization arising from the periodic boundary condition. ${ }^{52,53}$ In the $2 \mathrm{D}$ case, however, since such system-bath coupling effects are canceled out by superposing the coherences during $T_{1}$ and $T_{3}$ with respect to each energy level, we observe the discretization of energy levels as three distinct peaks parallel to the $\omega_{3}=\omega_{1}$ direction (diagonal di- 
rection), which reflect the transition processes shown in Figs. 2(d) $-2(\mathrm{f})$.

Notice that such three antidiagonal peaks are caused by the quantization of the angular momentum and they will vanish in the classical limit where the angular momentum is continuous.

By comparing Figs. 4(a-i), 4(a-ii), and 4(a-iii) with Figs. 4(b-i), 4(b-ii), and 4(b-iii), respectively, we find that the peaks parallel to the line $\omega_{1}=-\omega_{3}$ shift to the high frequency as temperature increases because the effects of the initial thermal distribution remain in the spectral line along the $\omega_{3}=-\omega_{1}$ direction (antidiagonal direction). Such temperature dependence of the peak shift was also observed in the $1 \mathrm{D}$ absorption spectra. ${ }^{53}$

For small $\gamma$ satisfying $\gamma \ll \alpha$ [Figs. 4(a-i) and 4(b-i)], the approximated expression of the third-order response, Eq. (2.6),

$$
\begin{aligned}
R^{(3)}\left(T_{3}, T_{2}=0, T_{1}\right) \\
\simeq-\frac{1}{\hbar^{3}} \sin ^{2}\left(\alpha T_{3}\right)\left[2 e^{-\left(T_{1}+T_{3}\right)^{2} /(2 \mu \beta)} \sin \left\{\alpha\left(T_{1}+T_{3}\right)\right\}\right. \\
\left.\quad+e^{-\left(-T_{1}+T_{3}\right)^{2} /(2 \mu \beta)} \sin \left\{\alpha\left(-T_{1}+T_{3}\right)\right\}\right],
\end{aligned}
$$

indicates the continuous broad band along the antidiagonal direction, which has been mentioned earlier. The temperature dependence of the linewidth along the antidiagonal direction is provided by the factor $e^{-\left(-T_{1}+T_{3}\right)^{2} /(2 \mu \beta)}$ in Eq. (3.2), because the contribution of the second term in square brackets is more dominant than the first term for $T_{1}, T_{3}>0$ due to the factor $e^{-\left( \pm T_{1}+T_{3}\right)^{2} /(2 \mu \beta)}$.

Comparing Figs. 4(a-ii) and 4(b-ii) with Figs. 4(a-i) and 4(b-i), respectively, we find that the linewidths along the $\omega_{3}=\omega_{1}$ direction (diagonal direction) become broader with increasing damping, because, in this direction, the effects of the initial thermal distribution are canceled out through the higher-order optical process and the damping effects become apparent. It should be emphasized again that the sensitivity of the 2D spectral line along the diagonal direction to the damping effect cannot be observed in 1D spectra (Fig. 3) in which the initial thermal distribution contributes more strongly than the damping effect.

Figures 4(a-iii) and 4(b-iii) show the colored noise case. In this case, the effective damping strength becomes weaker than in Figs. 4(a-ii) and 4(b-ii), because it is expressed as $|\gamma(\omega)|$ with $\gamma(\omega)=\gamma /\left(1-i \omega / \omega_{D}\right)$. Hence the linewidth along the diagonal direction in Fig. 4(a-iii) [Fig. 4(b-iii)] is narrower than that in Fig. 4(a-ii) [Fig. 4(b-ii)].

Next, we study the strongly damped case, where the damping effects are larger than quantum and thermal effects, i.e., $\gamma \gg \alpha$ and $\gamma \gg(\beta \hbar)^{-1}$. Figure 5 shows the $2 \mathrm{D}$ spectra for the strongly damped case for (a) $\omega_{D}=\infty$, i.e., the Ohmic dissipation, and (b) $\omega_{D}=0.1 \gamma$ for fixed $\gamma=1.3 \times 10^{14} \mathrm{~Hz}$ and $T=50 \mathrm{~K}$. In order to study the signals in detail, we consider them in section as in the right [Figs. $5\left(\mathrm{a}^{\prime}\right)$ and $5\left(\mathrm{~b}^{\prime}\right)$ ] and the above contour plot [Figs. $5\left(\mathrm{a}^{\prime \prime}\right)$ and $5\left(\mathrm{~b}^{\prime \prime}\right)$ ], which are the signals at $\omega_{1} / \alpha=16$ and at $\omega_{3} / \alpha=32$, respectively. For the Ohmic case [Fig. 5(a)], the peak at $\left(\omega_{1}, \omega_{3}\right)=(0,0)$ corre-
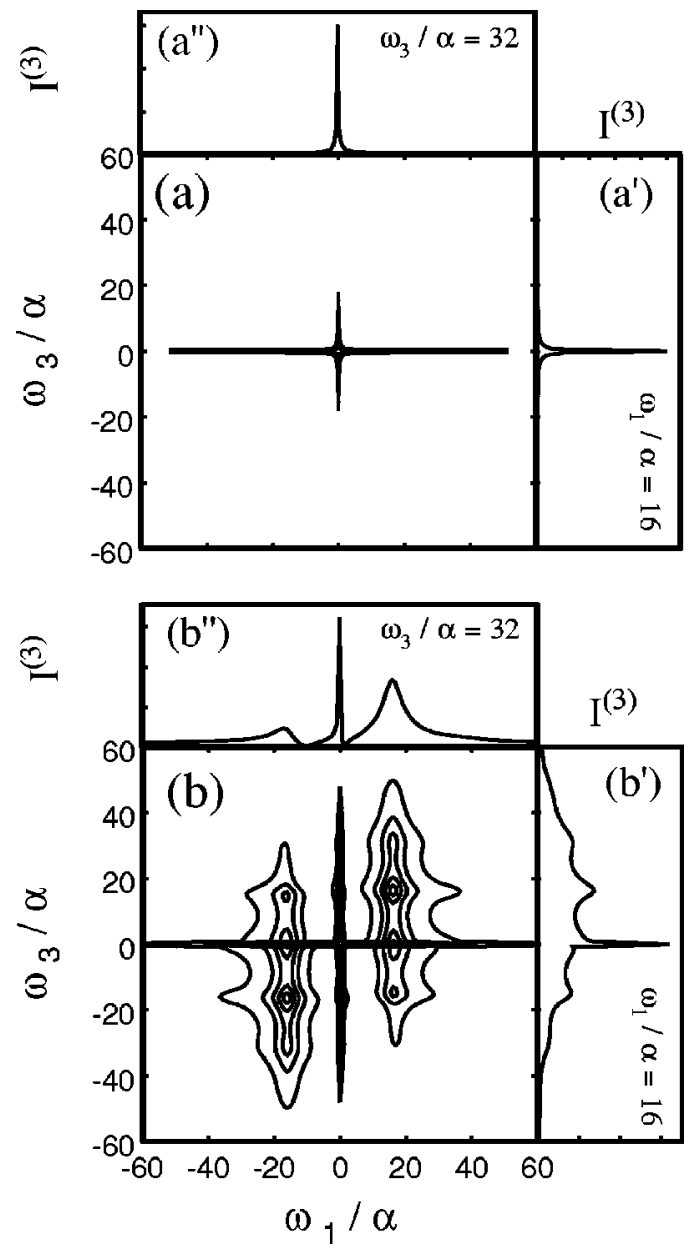

FIG. 5. Two-dimensional signal $I^{(3)}\left(\omega_{3}, \omega_{1}\right)$ for a strongly damped rotator for (a) $\omega_{D}=\infty$ and (b) $\omega_{D}=0.1 \gamma$, and for fixed $\gamma=1.3 \times 10^{14} \mathrm{~Hz}$ and $T$ $=50 \mathrm{~K}$. The right and the above of the contour plot, (b)-1 and (b)-2, are the signals at $\omega_{1} / \alpha=16$ and $\omega_{3} / \alpha=32$, respectively.

sponds to the strongly damped rotational motion as stated in Ref. 52.

For the case of the small cutoff frequency in the colored noise, satisfying $\omega_{D}<\gamma$ [Fig. 5(b)], we observe peaks at $\left(\omega_{1}, \omega_{3}\right)= \pm\left(\bar{\Omega}, n_{3} \bar{\Omega}\right) \quad\left(n_{3}=0, \pm 1, \pm 2, \pm 3\right) \quad$ and $\left(\omega_{1}, \omega_{3}\right)$ $=\left(0, n_{3}^{\prime} \bar{\Omega}\right) \quad\left(n_{3}^{\prime}=0, \pm 1, \pm 2\right)$, where $\bar{\Omega}=15.8 \alpha$, in addition to the peak at the origin. The shape of the $2 \mathrm{D}$ spectrum is different from that for the weakly damped rotator and similar to that for the vibrational motion.

Such features are interpreted as follows: Suppose that the frequency of the bath oscillators $\omega_{i}$ are distributed in the narrow range around some frequency $\omega_{0} \sim 0\left(\omega_{0} \neq 0\right)$ due to the small cutoff, the Hamiltonian (2.1) is approximated with a typical coordinate and conjugated momentum of the bath oscillator, $\hat{x}_{0}$ and $\hat{p}_{0}$, by

$$
\hat{H}=\frac{L^{2}}{2 \mu}+\frac{1}{2} \mu \bar{\Omega}^{2} \theta^{2}+\frac{\hat{p}_{0}^{2}}{2 \bar{m}}+\frac{1}{2} \bar{m} \bar{\omega}^{2} \hat{q}_{0}^{2}-\bar{c} \hat{q}_{0} \theta+\hat{H}^{\prime},
$$

where $\quad \bar{\Omega}=\sqrt{\gamma \omega_{D}}, \quad 1 / \bar{m}=\Sigma_{i}\left(1 / m_{i}\right), \quad \bar{\omega}^{2}=\sum m_{i} \omega_{i}^{2} / \bar{m}, \quad \bar{c}$ $=\Sigma_{i} c_{i}$, and $\hat{H}^{\prime}$ is the higher-order terms of $\left(\hat{q}_{i}-\hat{q}_{0}\right)$ or $\left(\hat{p}_{i}-\hat{p}_{0}\right)$ given by 


$$
\begin{aligned}
\hat{H}^{\prime}= & \sum_{i}\left[\frac{\left(\hat{p}_{i}-\hat{p}_{0}\right) \hat{p}_{0}}{m_{i}}+m_{i} \omega_{i}^{2}\left(\hat{q}_{i}-\hat{q}_{0}\right)\left(\hat{q}_{0}-\frac{c_{i} \theta}{m_{i} \omega_{i}^{2}}\right)\right] \\
& +\sum_{i}\left[\frac{\left(\hat{p}_{i}-\hat{p}_{0}\right)^{2}}{2 m_{i}}+\frac{m_{i} \omega_{i}^{2}}{2}\left(\hat{q}_{i}-\hat{q}_{0}\right)^{2}\right] .
\end{aligned}
$$

In Eq. (3.3), we have used the relation, $\int_{0}^{\infty} d \omega I(\omega) d \omega$ $=\Sigma_{i} c_{i}^{2} /\left(2 m_{i} \omega_{i}^{2}\right)=\mu \gamma \omega_{D} / 2$. The lowest order of $\left(\hat{q}_{i}-\hat{q}_{0}\right)$ or $\left(\hat{p}_{i}-\hat{p}_{0}\right)$ can be diagonalized,

$$
\hat{H} \sim \sum_{j=\mathrm{I}, \mathrm{II}}\left[\frac{p_{y_{j}^{2}}}{2 A \bar{m} \mu}+A \bar{m} \mu \lambda_{j}^{2} y_{j}^{2}\right],
$$

where $\quad A=\sqrt{\left(\bar{\Omega}^{2}-\bar{\omega}^{2}\right)^{2}+4 \bar{c}^{2} /(\mu \bar{m})} \quad$ and $\quad \lambda_{\mathrm{I}, \mathrm{II}}$ $=\sqrt{\left(\bar{\Omega}^{2}+\bar{\omega}^{2} \mp A\right) / 2}$. In Eq. (3.5), the normal modes I and II have been introduced as

$$
\begin{aligned}
& y_{\mathrm{I}}=f_{11} \theta+f_{12} \hat{q}_{0}, \\
& y_{\mathrm{II}}=f_{21} \theta+f_{22} \hat{q}_{0},
\end{aligned}
$$

and $p_{y_{j}}$ is the conjugated momentum of $y_{j}$, where the coefficients $f_{j k}(j, k=1,2)$ are given in Appendix C. For $\bar{\omega} / \bar{\Omega}$ $\ll 1$, the normal mode frequencies are approximately given by $\lambda_{\mathrm{I}} \sim 0\left(\lambda_{\mathrm{I}} \neq 0\right)$ and $\lambda_{\mathrm{II}} \sim \bar{\Omega}$. The coupling between $\hat{q}_{0}, \hat{p}_{0}$, $\theta$ and oscillators denoted by the coordinate $\left(\hat{q}_{i}-\hat{q}_{0}\right)$ and the momentum $\left(\hat{p}_{i}-\hat{p}_{0}\right)$ in the higher-order term $\hat{H}^{\prime}$ causes the damping of the normal modes $y_{\mathrm{I}}$ and $y_{\mathrm{II}}$. Modes I and II can be assigned to the overdamped motion and the damped vibrational motion, respectively.

Since the angular coordinate is expressed as $\theta$ $=2 A \bar{m}\left(f_{11} y_{\mathrm{I}}+f_{12} y_{\mathrm{II}}\right)$, the laser excitation with the dipole moment $d_{0} \cos \theta=d_{0} \cos \left[2 A \bar{m}\left(f_{11} y_{\mathrm{I}}+f_{12} y_{\mathrm{II}}\right)\right]$ causes multiquanta excitation-de-excitation processes for mode I and mode II described by $\left(y_{\mathrm{I}}\right)^{n_{1}}\left(y_{\mathrm{II}}\right)^{n_{2}} \quad\left(n_{1}, n_{2}=0\right.$, 1 , $\pm 2, \ldots)$. The peak positions can be understood in terms of diagrams similar to Fig. 2 . We consider the state $\left|n_{\mathrm{I}}, n_{\mathrm{II}}\right\rangle$ where $n_{\mathrm{I}}$ and $n_{\mathrm{II}}$ are the quantum number for modes I and II, respectively. The interaction between the laser and the system $\left(\propto d_{0} \cos \left[2 A \bar{m}\left(f_{11} y_{\mathrm{I}}+f_{12} y_{\mathrm{II}}\right)\right]\right)$ induces the transition $\left|n_{\mathrm{I}}, n_{\mathrm{II}}\right\rangle \quad$ to $\quad\left|n_{\mathrm{I}}+\Delta n_{\mathrm{I}}, n_{\mathrm{II}}+\Delta n_{\mathrm{II}}\right\rangle \quad\left(\Delta n_{\mathrm{I}}, \Delta n_{\mathrm{II}}=0, \pm 1\right.$, $\pm 2, \ldots)$. Such diagrams can lead to the peak position $\left(\omega_{1}, \omega_{3}\right)=\left(n_{1} \bar{\Omega}, n_{2} \bar{\Omega}\right)$, where $n_{1}$ and $n_{2}$ are integers. For example, the peak at the origin corresponds to the excitation-de-excitation process for mode I and the peaks at $\pm\left(\bar{\Omega}, n^{\prime} \bar{\Omega}\right) \quad(n=0, \pm 2)$ reveal the transition processes for mode II, which are originated from the coherence with the frequency $\bar{\Omega}$ in the time period $T_{1}$ and that with $2 \bar{\Omega}$ in the time period $T_{3}$. The peaks at $(0, n \bar{\Omega})(n= \pm 1, \pm 2)$ arise from the cross terms $\bar{\theta}_{\mathrm{I}} \bar{\theta}_{\mathrm{II}}, \bar{\theta}_{\mathrm{I}}^{2} \bar{\theta}_{\mathrm{II}}$, and $\bar{\theta}_{\mathrm{I}} \bar{\theta}_{\mathrm{II}}^{2}$ that are included in the dipole moment.

Notice that the two normal modes are observed in the absorption spectra; the dashed and dotted lines in Fig. 2 of Ref. 52. However, the 2D spectra give more detailed information on these peaks. In addition to the information for the overdamped mode and the vibrational mode, their coupling mechanism through the nonlinear dipole moment is revealed.
It should also be noted that we can observe two such normal modes in the strongly damped Brownian oscillator system for the small cutoff frequency. ${ }^{55}$

\section{CONCLUSION}

In this paper, we calculated the four-time correlation function for a damped two-dimensional rotator described by the Hamiltonian (2.1) from the generating functional obtained in Ref. 52. The result is fully quantum, however, and offers a clear physical insight into the relation between the classical and quantum regimes. Using this expression [Eqs. (2.5)-(2.8)], we plot 2D spectra corresponding to the farinfrared or microwave photon echo experiment in the case of Gaussian-Markovian noise for various coupling strengths, noise correlation times, and temperatures.

For a free rotator case, 2D spectra reveal three lines of discrete peaks which are parallel to the echo line $\omega_{3}$ $=-\omega_{1}$; each peak corresponds to a rotational level transition for a specific Liouville pathway. If we include the heatbath, these discrete peaks are merged into three lines of peaks. This is because, for this linear-linear system-bath coupling model, the effective mass of the rotator becomes essentially infinity due to the masses of bath oscillators. This effect reduces the energy differences between the peaks to infinitely small separations as discussed in the onedimensional case. ${ }^{53}$ Although the discrete peaks along the lines vanish, even for the weak damping case, we can still extract the quantum effects as the separation of three lines of peaks in the present two-dimensional measurement. This separation is possible because the linewidths along the $\omega_{3}$ $=-\omega_{1}$ direction depend on the temperature through the initial distribution, while the linewidths along the $\omega_{3}=\omega_{1}$ direction are not sensitive to the temperature but the damping because the effects of the initial thermal distribution involved in the Liouville paths are canceled out in this higher-order optical process. When the damping becomes strong, the three lines of peaks are broadened and merge to a featureless peak. If the noise correlation time is very long for the strongly damped case, however, the signals exhibit a very different behavior. We observe an overdamped peak and an oscillatory peak and their cross peaks. These peaks can be related to two normal modes, an overdamped mode and vibrational one, arising from the collective motion of the free rotator and bath oscillators, in which the bath oscillators behave like a single mode oscillator due to the narrow spectral width. Such features can be seen in the 1D case, but 2D spectra more clearly indicate the origin of these two peaks.

In the present study, we focused on the analysis of the single rotator system linearly coupled with the heat-bath. Although this model reduces to the Langevin equation in the classical limit, this is by no means the only model as a damped rotator system. An alternative model will be to consider different system-bath couplings, which satisfies the cyclic boundary condition of the rotator coordinate without tracing over the heat-bath degrees of freedom. Generalization to a three-dimensional rotator system is also important for some molecules at low temperatures where the quantum effects play a role. ${ }^{56}$ To account for experiment results, such as the infrared optical response of dielectric molecular liquids 
or peptides, one may need to consider many rotators interacting with each other through dipole-dipole interactions. Comparison between the results from a model calculation and molecular dynamics simulation are also important to justify the validity of the model. Critical checks for the validity of the model may be done by comparing calculated twodimensional signals with experimental studies. Such comparison would help quite the proper description of the coupling to the bath and the dipolar interaction.

\section{ACKNOWLEDGMENTS}

We wish to thank Tsuyoshi Kato for useful discussions. Y.T. thanks financial support of a Grant-in-Aid for Scientific Research (B) (12440171) from Japan Society for the Promotion of Science and Morino Science Foundation.

\section{APPENDIX A: MULTITIME CORRELATION FUNCTIONS AND THEIR GENERATING FUNCTIONAL}

In this Appendix, we present the higher-order response function in the two-dimensional rotator system coupled to a heat bath. Consider the generating functional $Z[K]$ defined as

$$
Z[K]=\operatorname{Tr}\left(\hat{\rho}_{I}^{K_{3}} \hat{U}_{K_{2}}^{\dagger}\left(\infty, t_{I}\right) \hat{U}_{K_{1}}\left(\infty, t_{I}\right)\right),
$$

where

$$
\hat{U}_{K_{\alpha}}\left(\infty, t_{I}\right)=\mathrm{T}_{t}\left(\exp \left(-\frac{i}{\hbar} \int_{t_{I}}^{\infty} d t\left(\hat{H}-K_{\alpha}(t) \cos \theta\right)\right)\right)
$$

and

$$
\hat{\rho}_{I}^{K_{3}}=\mathrm{T}_{\tau}\left(\exp \left(-\frac{1}{\hbar} \int_{0}^{\beta \hbar} d \tau\left(\hat{H}-\frac{i}{\hbar} K_{3}(\tau) \cos \theta\right)\right)\right) .
$$

Here, $\hat{\rho}_{I}{ }^{K}=0$ gives an equilibrium distribution at the initial time $t_{I}$ and $\tau$ is the imaginary time variable with $0 \leqslant \tau$ $\leqslant \beta \hbar$ in which $\beta$ is the inverse temperature. The symbol $\mathrm{T}_{t(\tau)}$ stands for the real (imaginary) time ordering operator. We represent the function in the two real time path by the suffix $\alpha=1,2$ and the imaginary one by $\alpha=3$, respectively.

From Ref. 52, the functional $Z[K]$ is rewritten in terms of the new functional $\bar{Z}[J]$ :

$$
\begin{aligned}
Z[K]= & \bar{Z}[J=0]+\sum_{N=1}^{\infty} \sum_{a_{1}= \pm 1} \cdots \sum_{a_{N}= \pm 1} \int_{C} d t_{1} \cdots \int_{C} d t_{N} \\
& \times \frac{K\left(t_{1}\right) \cdots K\left(t_{N}\right)}{N ! 2^{N}}(\bar{Z}[J])_{J(s) / \hbar=\Sigma_{i=1}^{N} a_{i} \delta_{C}\left(s-t_{i}\right)}
\end{aligned}
$$

Here $\bar{Z}[J]$ is given by

$$
\begin{aligned}
\bar{Z}[J]= & \frac{\bar{Z}[J=0]}{\Lambda} \delta_{0, R_{J}} e^{\Xi[J]} \sum_{l} \exp \left(-\frac{1}{\beta \hbar^{2}}[-2 \pi l\right. \\
& \left.\left.\times \int_{C} d s\left(t_{I}-i \beta \hbar-s\right) J(s)+\frac{\mu^{\prime}}{2}(2 \pi l)^{2}\right]\right),
\end{aligned}
$$

where $R_{J}=\int_{C} d t J(t) / \hbar, \Lambda=\Sigma_{l} e^{-\mu^{\prime}(2 \pi l)^{2} /\left(2 \beta \hbar^{2}\right)}$, and the in$\operatorname{dex} C$ in the integral, $\int_{C} d t$, implies the integration along the contour time path that starts from $t_{I}$ to $\infty$ along the real path $\left(C_{1}\right)$, returns to $t_{I}\left(C_{2}\right)$ and goes to $t_{I}-i \beta \hbar$ parallel to the imaginary axis $\left(C_{3}\right) \cdot{ }^{57,58}$ The condition $R_{J}=0$ in Eq. (A5) selects energy level transitions induced by irradiated pulses. The function $\Xi[J]$ is given by

$$
\begin{aligned}
\Xi[J] \equiv & \frac{i}{2 \hbar} \int_{t_{I}}^{\infty} d t \int_{t_{I}}^{\infty} d t^{\prime}\left(2 J_{-}(t) K_{0}^{(+-)}\left(t-t^{\prime}\right) J_{+}\left(t^{\prime}\right)\right. \\
& \left.+J_{-}(t) K_{0}^{(++)}\left(t-t^{\prime}\right) J_{-}\left(t^{\prime}\right)\right) \\
& +\frac{1}{\hbar} \int_{t_{I}}^{\infty} d t \int_{0}^{\beta \hbar} d \tau J_{-}(t) K_{0}^{(+3)}(t, \tau) J_{3}(\tau) \\
& -\frac{i}{2 \hbar} \int_{0}^{\beta \hbar} d \tau \int_{0}^{\beta \hbar} d \tau^{\prime} J_{3}(\tau) K_{0}^{(33)}\left(\tau-\tau^{\prime}\right) J_{3}\left(\tau^{\prime}\right)
\end{aligned}
$$

where $J_{+} \equiv\left(J_{1}+J_{2}\right) / 2, \quad J_{-} \equiv J_{1}-J_{2}$. The functions $K_{0}^{(+-)}(t)$ and $K_{0}^{(++)}(t)$ are represented as in Eqs. (2.9) and (2.11), respectively. The Fourier-Laplace transform of $K_{0}^{(+3)}(t, \tau)$ and the Fourier transform of $K_{0}^{(33)}(\tau)$ are denoted as

$$
\begin{aligned}
& K_{0}^{(+3)}\left[z, \nu_{n}\right]=\frac{i}{z+\nu_{n}}\left(K_{0}^{(+-)}\left[\nu_{n}\right]-K_{0}^{(+-)}[z]\right) . \\
& K_{0}^{(33)}\left[\nu_{n}\right]=i K_{0}^{(+-)}\left[-\nu_{n}\right] .
\end{aligned}
$$

The $N+1$-time correlation function of $\cos \theta$ is evaluated from the generating functional as

$$
\begin{aligned}
& \left\langle\mathrm{T}_{C} \cos \hat{\theta}\left(t_{0}\right) \cos \hat{\theta}\left(t_{1}\right) \cdots \cos \hat{\theta}\left(t_{N}\right)\right\rangle \\
& \quad=\left.\frac{1}{Z[K=0]}\left(\frac{\hbar}{i}\right)^{N+1} \frac{\delta^{N+1} Z[K]}{\delta_{C} K\left(t_{0}\right) \delta_{C} K\left(t_{1}\right) \cdots \delta_{C} K\left(t_{N}\right)}\right|_{K=0} .
\end{aligned}
$$

Here, the index $C$ implies the contour time path. The operator $T_{C}$ and the function $\delta_{C}(t)$ are the time-ordering operator and the $\delta$ function on the contour time path, respectively. The functional differentiation $\delta / \delta_{C} K(t)$ means $\delta / \delta K_{1}(t)$, $-\delta / \delta K_{2}(t)$, and $(\hbar / i) \delta / \delta K_{3}(t)$ for $t \in C_{1}, t \in C_{2}$, and $t$ $\in C_{3}$. From Eqs. (A4) and (A9), the $(N+1)$-time correlation function is expressed as 


$$
\left\langle\mathrm{T}_{C} \cos \theta\left(t_{0}\right) \cos \theta\left(t_{1}\right) \cdots \cos \theta\left(t_{N}\right)\right\rangle=\left.\sum_{a_{0}= \pm 1} \cdots \sum_{a_{N}= \pm 1} \frac{\bar{Z}[J]}{2^{N+1} \bar{Z}[J=0]}\right|_{J(s)=\hbar\left(a_{0} \delta_{C}\left(s-t_{0}\right)+a_{1} \delta_{C}\left(s-t_{1}\right)+\cdots+a_{N} \delta_{C}\left(s-t_{N}\right)\right)} .
$$

Notice that the correlation function (A10) vanishes when $N$ is even due to the Kronecker delta $\delta_{0, R_{J}}$, which implies the rotational symmetry of the rotator. From Eqs. (2.4) and (A9), the $N$ th-order response function is given by the differentiation of the generating functional $Z[K]$ as follows:

$$
\begin{aligned}
R^{(N)}( & \left.T_{N}, \ldots, T_{2}, T_{1}, T_{2}, T_{1}\right) \\
= & \theta\left(T_{1}\right) \theta\left(T_{2}\right) \cdots \theta\left(T_{N}\right) \\
& \times \frac{\hbar}{i} \cdot \frac{1}{Z[K=0]}\left(\frac{\delta^{N+1} Z[K]}{\delta K_{-}\left(t_{0}\right) \delta K_{+}\left(t_{1}\right) \cdots \delta K_{+}\left(t_{N}\right)}\right)_{K=0},
\end{aligned}
$$$$
R^{(N)}\left(T_{N}, \ldots, T_{2}, T_{1}\right)
$$$$
=\theta\left(T_{1}\right) \theta\left(T_{2}\right) \cdots \theta\left(T_{N}\right)\left(\frac{i}{\hbar}\right)^{N}\left(\prod_{i=0}^{N} \sum_{c_{i}=1,2}(-1)^{c_{i}+1}\right)
$$$$
\times\left.\left(\prod_{j=0}^{N} \sum_{a_{j}= \pm 1}\right) \frac{(-1)^{c_{0}+1} \bar{Z}[J]}{2^{N+2} \bar{Z}[J=0]}\right|_{J(s)=\hbar \Sigma_{i=0}^{N} a_{i} \delta_{C_{c_{i}}}\left(s-t_{i}\right)},
$$

(A11)

where $\quad K_{+}(t) \equiv\left(K_{1}(t)+K_{2}(t)\right) / 2 \quad$ and $\quad K_{-}(t) \equiv K_{1}(t)$ $-K_{2}(t)$ and we set $t_{i}=T_{1}+T_{2}+\cdots+T_{N-i}$ (for $i$ $=0,1, \ldots, N-1)$ and $t_{N}=0$ as in Sec. II. With the use of Eqs. (A9) and (A10), Eq. (A11) is expressed as where $\delta_{C_{c_{i}}}\left(s-t_{i}\right)$ is the delta function on the counter time path satisfying the condition $t_{i} \in C_{c_{i}}$. Equation (A12) gives the analytical expression of the $N$ th order response function.

\section{APPENDIX B: THE EXPRESSION OF THE FUNCTIONS $\boldsymbol{R}_{A}, \boldsymbol{R}_{B}$, AND $\boldsymbol{R}_{C}$ IN EQ. (2.5)}

The functions $R_{A}, R_{B}$, and $R_{C}$ in Eq. (2.5) are expressed as follows:

$$
\begin{aligned}
R_{A}^{(3)}\left(T_{3}, T_{2}, T_{1}\right)= & \sum_{l}\left\{\exp \left(-\frac{\mu^{\prime}(2 \pi l)^{2}}{2 \beta \hbar^{2}}\right) \cosh \left[\frac{2 \pi l}{\beta \hbar}\left(t_{0}+t_{1}-t_{2}-t_{3}\right)\right]\right\} \\
& \times \exp \left[i \hbar \left(\bar{K}_{0}^{(++)}\left(t_{0}-t_{1}\right)-\bar{K}_{0}^{(++)}\left(t_{0}-t_{2}\right)-\bar{K}_{0}^{(++)}\left(t_{0}-t_{3}\right)-\bar{K}_{0}^{(++)}\left(t_{1}-t_{2}\right)-\bar{K}_{0}^{(++)}\left(t_{1}-t_{3}\right)\right.\right. \\
& \left.\left.+\bar{K}_{0}^{(++)}\left(t_{2}-t_{3}\right)\right)\right] \sin \left(\frac{\hbar}{2} K_{0}^{(+-)}\left(t_{0}-t_{1}\right)\right) \sin \left[\frac{\hbar}{2}\left(K_{0}^{(+-)}\left(t_{0}-t_{2}\right)+K_{0}^{(+-)}\left(t_{1}-t_{2}\right)\right)\right] \\
& \times \sin \left[\frac{\hbar}{2}\left(K_{0}^{(+-)}\left(t_{0}-t_{3}\right)+K_{0}^{(+-)}\left(t_{1}-t_{3}\right)-K_{0}^{(+-)}\left(t_{2}-t_{3}\right)\right)\right], \\
R_{B}^{(3)}\left(T_{3}, T_{2}, T_{1}\right)= & \sum_{l}\left\{\exp \left(-\frac{\mu^{\prime}(2 \pi l)^{2}}{2 \beta \hbar^{2}}\right) \cosh \left[\frac{2 \pi l}{\beta \hbar}\left(t_{0}-t_{1}+t_{2}-t_{3}\right)\right]\right\} \\
& \times \exp \left[i \hbar \left(-\bar{K}_{0}^{(++)}\left(t_{0}-t_{1}\right)+\bar{K}_{0}^{(++)}\left(t_{0}-t_{2}\right)-\bar{K}_{0}^{(++)}\left(t_{0}-t_{3}\right)-\bar{K}_{0}^{(++)}\left(t_{1}-t_{2}\right)+\bar{K}_{0}^{(++)}\left(t_{1}-t_{3}\right)\right.\right. \\
& \left.\left.-\bar{K}_{0}^{(++)}\left(t_{2}-t_{3}\right)\right)\right] \sin \left(\frac{\hbar}{2} K_{0}^{(+-)}\left(t_{0}-t_{1}\right)\right) \sin \left[\frac{\hbar}{2}\left(K_{0}^{(+-)}\left(t_{0}-t_{2}\right)-K_{0}^{(+-)}\left(t_{1}-t_{2}\right)\right)\right] \\
& \times \sin \left[\frac{\hbar}{2}\left(K_{0}^{(+-)}\left(t_{0}-t_{3}\right)-K_{0}^{(+-)}\left(t_{1}-t_{3}\right)+K_{0}^{(+-)}\left(t_{2}-t_{3}\right)\right)\right],
\end{aligned}
$$




$$
\begin{aligned}
R_{C}^{(3)}\left(T_{3}, T_{2}, T_{1}\right)= & \sum_{l}\left\{\exp \left(-\frac{\mu^{\prime}(2 \pi l)^{2}}{2 \beta \hbar^{2}}\right) \cosh \left[\frac{2 \pi l}{\beta \hbar}\left(t_{0}-t_{1}-t_{2}+t_{3}\right)\right]\right\} \\
& \times \exp \left[i \hbar \left(-\bar{K}_{0}^{(++)}\left(t_{0}-t_{1}\right)-\bar{K}_{0}^{(++)}\left(t_{0}-t_{2}\right)+\bar{K}_{0}^{(++)}\left(t_{0}-t_{3}\right)+\bar{K}_{0}^{(++)}\left(t_{1}-t_{2}\right)-\bar{K}_{0}^{(++)}\left(t_{1}-t_{3}\right)\right.\right. \\
& \left.\left.-\bar{K}_{0}^{(++)}\left(t_{2}-t_{3}\right)\right)\right] \sin \left(\frac{\hbar}{2} K_{0}^{(+-)}\left(t_{0}-t_{1}\right)\right) \sin \left[\frac{\hbar}{2}\left(K_{0}^{(+-)}\left(t_{0}-t_{2}\right)-K_{0}^{(+-)}\left(t_{1}-t_{2}\right)\right)\right] \\
& \times \sin \left[\frac{\hbar}{2}\left(K_{0}^{(+-)}\left(t_{0}-t_{3}\right)-K_{0}^{(+-)}\left(t_{1}-t_{3}\right)-K_{0}^{(+-)}\left(t_{2}-t_{3}\right)\right)\right]
\end{aligned}
$$

where $K^{(+-)}$is given by Eq. (2.9) and $\bar{K}^{(++)}$is defined by Eqs. (2.10) and (2.11).

Note that the functions $R_{A}^{(3)}, R_{B}^{(3)}$, and $R_{C}^{(3)}$ correspond to the terms in the third-order response:

$$
\begin{aligned}
& \left\langle\left[\left[\left[e^{i \theta\left(t_{0}\right)}, e^{i \theta\left(t_{1}\right)}\right], e^{-i \theta\left(t_{2}\right)}\right], e^{-i \theta\left(t_{3}\right)}\right]\right\rangle \\
& \quad+(\text { complex conjugate }(\text { c.c. })), \\
& \left\langle\left[\left[\left[e^{i \theta\left(t_{0}\right)}, e^{-i \theta\left(t_{1}\right)}\right], e^{i \theta\left(t_{2}\right)}\right], e^{-i \theta\left(t_{3}\right)}\right]\right\rangle+\text { (c.c.), } \\
& \left\langle\left[\left[\left[e^{i \theta\left(t_{0}\right)}, e^{-i \theta\left(t_{1}\right)}\right], e^{-i \theta\left(t_{2}\right)}\right], e^{i \theta\left(t_{3}\right)}\right]\right\rangle+\text { (c.c.) },
\end{aligned}
$$

respectively, so that the Feynman diagrams, Figs. 2(a),2(c), Fig. 2(b), and Figs. 2(d)-2(f) can be associated with the functions $R_{A}^{(3)}, R_{B}^{(3)}$, and $R_{C}^{(3)}$.

\section{APPENDIX C: COEFFICIENTS IN DEFINITION OF TWO NORMAL MODES}

In Eqs. (3.6) and (3.7), the coefficients $f_{j k}(j, k=1,2)$ are given by

$$
\begin{aligned}
& f_{11}=-\frac{1}{2 A} \sqrt{\frac{A+\bar{\Omega}^{2}-\bar{\omega}^{2}}{\bar{m}}}, \\
& f_{12}=\frac{1}{2 A} \sqrt{\frac{A-\bar{\Omega}^{2}+\bar{\omega}^{2}}{\mu}}, \\
& f_{21}=\frac{1}{2 A} \sqrt{\frac{A-\bar{\Omega}^{2}+\bar{\omega}^{2}}{\bar{m}}}, \\
& f_{22}=\frac{1}{2 A} \sqrt{\frac{A+\bar{\Omega}^{2}-\bar{\omega}^{2}}{\mu}},
\end{aligned}
$$

where $A$ is defined by $A=\sqrt{\left(\bar{\Omega}^{2}-\bar{\omega}^{2}\right)^{2}+4 \bar{c}^{2} /(\mu \bar{m})}$, as stated in Sec. III.

${ }^{1}$ S. Mukamel, Principles of Nonlinear Optical Spectroscopy (Oxford University Press, New York, 1995).

${ }^{2}$ Y. Tanimura and S. Mukamel, J. Chem. Phys. 99, 9496 (1993).

${ }^{3}$ K. Okumura and Y. Tanimura, J. Chem. Phys. 106, 1687 (1997); 107, 2267 (1997); Chem. Phys. Lett. 277, 159 (1997); Y. Tanimura, Chem. Phys. 233, 217 (1998).

${ }^{4}$ Y. Tanimura, Proceedings of Two-Dimensional Correlation Spectroscopy, edited by Y. Ozaki (American Institute of Physics, New York, 2000), pp. $144-153$.

${ }^{5}$ K. Okumura and Y. Tanimura, Chem. Phys. Lett. 278, 175 (1997).

${ }^{6}$ M. Cho, K. Okumura, and Y. Tanimura, J. Chem. Phys. 108, 1326 (1998).
${ }^{7}$ M. Cho, J. Chem. Phys. 109, 5327 (1998); 109, 6627 (1998); J. Sung and M. Cho, ibid. 113, 7072 (2000).

${ }^{8}$ A. Ma and R. M. Stratt, Phys. Rev. Lett. 85, 1004 (2000); J. Chem. Phys. 116, 4962 (2002); 116, 4972 (2002).

${ }^{9}$ S. Saito and I. Ohmine, J. Chem. Phys. 108, 240 (1998); 106, 4889 (1997).

${ }^{10}$ S. Saito and I. Ohmine, Phys. Rev. Lett. 88, 207401 (2002).

${ }^{11}$ K. Okumura, A. Tokmakoff, and Y. Tanimura, J. Chem. Phys. 111, 492 (1999)

${ }^{12}$ M. Cho, Advances in Multi-photon Processes and Spectroscopy, edited by Y. Fujimura and S. H. Lin (World Scientific, Singapore, 1999), Vol. 12, pp. $1-71$.

${ }^{13}$ M. Cho, PhysChemComm 7, 1 (2002).

${ }^{14}$ K. Tominaga and H. Maekawa, Bull. Chem. Soc. Jpn. 74, 279 (2001).

${ }^{15}$ M. Khalil and A. Tokmakoff, Chem. Phys. 266, 213 (2001).

${ }^{16}$ C. Scheurer, A. Piryatinski, and S. Mukamel, J. Am. Chem. Soc. 123, 3114 (2001); A. Piryatinski, S. Tretiak, V. Chernyak, and S. Mukamel, J. Raman Spectrosc. 31, 125 (2000)

${ }^{17}$ R. Akiyama and R. F. Loring, J. Chem. Phys. 116, 4655 (2002).

${ }^{18}$ T. Steffen and Y. Tanimura, J. Phys. Soc. Jpn. 69, 3115 (2000).

${ }^{19}$ Y. Tanimura and T. Steffen, J. Phys. Soc. Jpn. 69, 4095 (2000).

${ }^{20}$ T. Kato and Y. Tanimura, J. Chem. Phys. 117, 6221 (2002); T. Kato and Y. Tanimura, ibid. (to be published).

${ }^{21}$ Y. Suzuki and Y. Tanimura, J. Chem. Phys. 115, 2267 (2001).

${ }^{22}$ V. Astinov, K. J. Kubarych, and C. J. Milne, R. J. D. Miller, J. Chem. Phys. 327, 3334 (2000); K. J. Kubarych, C. L. Milne, S. Lin, V. Astinov, and J. D. Miller, J. Chem. Phys. 116, 2016 (2002); K. J. Kubarych, C. L. Milne, S. Lin, and J. D. Miller, Appl. Phys. B: Lasers Opt. 74, S107 (2002).

${ }^{23}$ O. Golonzka, N. Demirdöven, M. Khalil, and A. Tokmakoff, J. Chem. Phys. 113, 9893 (2000).

${ }^{24}$ L. J. Kaufman, J. Heo, L. D. Ziegler, and G. R. Fleming, Phys. Rev. Lett. 88, 207402 (2002); L. J. Kaufman, D. A. Blank, and G. R. Fleming, J. Chem. Phys. 114, 2312 (2001); D. A. Blank, L. J. Kaufman, and G. R. Fleming, ibid. 113, 771 (2000); 111, 3105 (1999).

${ }^{25}$ J. C. Kirkwood and A. C. Albrecht, J. Raman Spectrosc. 31, 107 (2000); J. C. Kirkwood, A. C. Albrecht, and D. J. Ulness, J. Chem. Phys. 111, 253 (1999); J. C. Kirkwood, A. C. Albrecht, D. J. Ulness, and M. J. Stimson, ibid. 111, 272 (1999).

${ }^{26}$ M. Cho, D. A. Blank, J. Sung, K. Park, S. Hahn, and G. R. Fleming, J. Chem. Phys. 112, 2082 (2000).

${ }^{27}$ M. Cho, C. Hess, and M. Bonn, Phys. Rev. B 65, 205423 (2002).

${ }^{28}$ R. F. Loring and S. Mukamel, J. Chem. Phys. 83, 2116 (1985).

${ }^{29}$ M. Berg and D. A. Vanden Bout, Acc. Chem. Res. 30, 65 (1997); D. Vanden Baut, J. E. Freitas, and M. Berg, Chem. Phys. Lett. 229, 97 (1994).

${ }^{30}$ K. Tominaga and K. Yoshihara, Prog. Cryst. Growth Charact. Mater. 33, 371 (1996).

${ }^{31}$ K. Tominaga, G. P. Keogh, and K. Yoshihara, J. Mol. Liq. 65-66, 389 (1995).

${ }^{32}$ S. Gnanakaran and R. M. Hochstrasser, J. Am. Chem. Soc. 123, 12886 (2001).

${ }^{33}$ P. Hamm, M. Lim, and R. M. Hochstrasser, J. Phys. Chem. B 102, 6123 (1998).

${ }^{34}$ S. Woutersen and P. Hamm, J. Chem. Phys. 115, 7737 (2001); J. Phys. Chem. B 104, 11316 (2002).

${ }^{35}$ O. Golonzka, M. Kahlil, N. Demirdöven, and A. Tokmakoff, J. Chem. Phys. 115, 10814 (2001); N. N. Demirdöven, M. Kahlil, O. Golonzka, and A. Tokmakoff, J. Phys. Chem. A 105, 8025 (2001); O. Golonzka, M. Kahlil, N. Demirdiöven, and A. Tokmakoff, Phys. Rev. Lett. 86, 2154 (2001).

${ }^{36}$ L. Lepetit and M. Joffere, Opt. Lett. 85, 287 (2000). 
${ }^{37}$ W. Zhao and J. C. Wright, Phys. Rev. Lett. 83, 1950 (1999); 84, 1411 (2000); J. Am. Chem. Soc. 121, 10994 (1999).

${ }^{38}$ J. C. Wright, Int. Rev. Phys. Chem. 21, 185 (2002).

${ }^{39}$ R. A. Denny and D. R. Reichman, Phys. Rev. E 63, 065101 (2001); J. Chem. Phys. 116, 1979 (2002); 116, 1987 (2002).

${ }^{40}$ J. Cao, J. Wu, and S. Yang, J. Chem. Phys. 116, 3739 (2002); 116, 3760 (2002).

${ }^{41}$ T. I. C. Jansen, J. G. Snijders, and K. Duppen, J. Chem. Phys. 113, 307 (2000); 114, 10910 (2001)

${ }^{42}$ R. L. Murry, J. T. Fourkas, and T. Keyes, J. Chem. Phys. 109, 2814 (1998); 109, 7913 (1998); J. T. Fourkas and T. Keyes, ibid. 112, 287 (1998).

${ }^{43}$ J. Kim and T. Keyes, Phys. Rev. E 65, 061102 (2002).

${ }^{44}$ A. O. Caldeira and A. J. Leggett, Physica A 121, 587 (1983); 130, 374(E) (1985).

${ }^{45}$ H. Grabert, P. Schramm, and G.-L. Ingold, Phys. Rep. 168, 115 (1988).

${ }^{46}$ P. Hänggi, P. Talkner, and M. Borkovec, Rev. Mod. Phys. 62, 745 (1990).

${ }^{47}$ U. Weiss, Quantum Dissipative Systems, 2nd ed. (World Scientific, Singapore, 1999).
${ }^{48}$ J. L. Skinner and D. Hsu, Adv. Chem. Phys. 65, 1 (1986); J. Phys. Chem. 90, 4931 (1986); Chem. Phys. 128, 35 (1988).

${ }^{49}$ P. Debye, Polar Molecules (Reinhold, New York, 1929).

${ }^{50}$ Molecular Spectroscopy, edited by D. A. Long, D. J. Millen, and R. F. Barrow (Chemical Society, London, 1974), Vol. 2.

${ }^{51}$ W. G. Rothschild, Dynamics of Molecular Liquids (Wiley, New York, 1984).

${ }^{52}$ Y. Suzuki and Y. Tanimura, J. Phys. Soc. Jpn. 71, 2414 (2002).

${ }^{53}$ Y. Suzuki and Y. Tanimura, J. Phys. Soc. Jpn. 70, 1161 (2001).

${ }^{54}$ Y. Tanimura and R. Kubo, J. Phys. Soc. Jpn. 58, 101 (1989).

${ }^{55}$ Y. Suzuki and Y. Tanimura, Chem. Phys. Lett. 358, 51 (2002).

${ }^{56}$ J. Jang and R. M. Stratt, J. Chem. Phys. 113, 5901 (2000); 113, 11212 (2000).

${ }^{57}$ R. Fukuda, M. Sumino, and K. Nomoto, Phys. Rev. A 45, 3559 (1992).

${ }^{58}$ R. Fukuda, M. Komachiya, S. Yokojima, Y. Suzuki, K. Okumura, and T. Inagaki, Novel Use of Legendre Transformation in Field Theory and Many Particle Systems-On-Shell Expansion and Inversion Method [Prog. Theor. Phys. Suppl. 121, 1 (1995)]. 